\title{
UNA EVALUACIÓN PRELIMINAR DE LA HERPETOFAUNA EN LA ZONA RESERVADA ALLPAHUAYO - MISHANA, LORETO, PERÚ*
}

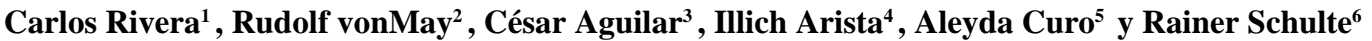

\begin{abstract}
RESUMEN
Fueron muestreados los anfibios y reptiles en la Zona Reservada Allpahuayo - Mishana, Loreto, Perú, entre los días 18 al 22 de noviembre del 2000, en el marco del curso "Uso de inventarios biológicos para revelar patrones de distribución geográfica de especies en la selva baja amazónica", organizado por el proyecto Diversidad Biológica de la Amazonía Peruana, Perú - Finlandia (BIODAMAZ), con el apoyo del Instituto de Investigaciones de la Amazonía Peruana (IIAP) y la Universidad Nacional de la Amazonía Peruana (UNAP). Fue registrado un total de 66 especies, de las cuales 49 fueron anfibios ( 22 géneros) y 17 reptiles (14 géneros). Seis especies de anuros fueron registradas por primera vez para la Zona Reservada, de las cuales tres especies (una Hyla y dos Eleutherodactylus) posiblemente serían nuevas para la Amazonía peruana. Las comparaciones de la composición y abundancia de especies entre los dos tipos de formaciones vegetales revelan diferencias considerables. Se presenta una lista completa de las especies registradas en las áreas estudiadas.
\end{abstract}

Palabras clave: Abundancia, Amazonía peruana, anfibios, composición, reptiles, Zona Reservada Allpahuayo - Mishana.

\begin{abstract}
Amphibians and reptiles were studied in the Allpahuayo-Mishana Reserved Zone, Loreto, Peru, between 18-22 November 2002, as part of the "Use of biological inventories to reveal geographic distribution patterns of lowland Amazonian species" course organized by the Peru-Finland Biological Diversity of the Peruvian Amazon project (BIODAMAZ), with the support of the Peruvian Amazonian Research Institute (IIAP) and the Peruvian Amazonian National University (UNAP). A total of 66 species were registered, of which 49 were amphibians (22 genera) and 17 reptiles (14 genera). Six anuran species were registered for the first time in the Reserved Zone. Of these, three species (one Hyla and two Eleutherodactylus) may be new for the Peruvian Amazon. Comparison of species composition and abundance between the two different forest types shows considerable differences. A complete list of the species found in the study area is presented.
\end{abstract}

Key words: Abundance, Allpahuayo-Mishana Reserved Zone, amphibians, composition, Peruvian Amazon, reptiles.

\section{INTRODUCCIÓN}

En pocos lugares del Neotrópico existe información completa, o casi completa, sobre la composición de especies de herpetofauna (algunos ejemplos: Crump, 1974; Duellman, 1978, 1990; Dixon y Soini, 1986; Rodríguez y Cadle 1990; Duellman y Salas, 1991; Rodríguez, 1992; Duellman y Mendelson III, 1995), a pesar de que éste

* Referencia bibliográfica del artículo: Rivera, C., vonMay, R, Aguilar, C., Arista, I., Curo, A. \& Schulte, R. 2002. Una evaluación preliminar de la herpetofauna en la Zona Reservada Allpahuayo - Mishana, Loreto, Perú. En: Rodríguez, J.J., Ruokolainen, K. \& Soini, P. (eds.). La diversidad biológica de la Zona Reservada Allpahuayo - Mishana, Loreto, Perú: Relaciones entre distribución, abundancia y hábitats. Folia Amazónica 14 (1-2): NÚMEROS DE PÁGINA.

1 Universidad Nacional de la Amazonía Peruana (UNAP), Iquitos, Perú. Correo electrónico: biodamaz@iiap.org.pe

2 Tambopata Research Center, Rainforest Expeditions, Madre de Dios, Perú.

3 Museo de Historia Natural, Universidad Nacional Mayor de San Marcos (UNMSM), Lima, Perú.

4 Universidad Nacional de la Amazonía Peruana (UNAP), Iquitos, Perú.

5 Universidad Nacional San Antonio Abad del Cusco (UNSAAC), Cusco, Perú.

6 INIBICO, Tarapoto, Perú. 
es un componente de gran importancia dentro de la diversidad biológica en un área. Esta información, entre otras, puede servir de base para diversos estudios de monitoreo de poblaciones, comparaciones entre hábitats y para determinar patrones de asociación con otros grupos de flora y fauna.

Adicionalmente, desde hace poco más de dos décadas se ha evidenciado una declinación de poblaciones de varias especies de anfibios en diversas partes del mundo (Wake y Morowitz, 1991), aún en áreas naturales protegidas, lo cual fue atribuido principalmente a factores como el cambio climático global y a patógenos (Berger et al., 1989; Donnelly y Crump, 1998). Para el Perú se tienen pocos datos relacionados con este problema global, por lo que son importantes no sólo datos de inventarios, sino también datos sobre la abundancia absoluta y relativa de diferentes poblaciones de anfibios (Vial y Saylor, 1993, citado en Salas et al., n.d.). Por otro lado, muchas especies de anfibios y reptiles se relacionan a hábitats específicos, aunque esto se ha determinado en pocos casos (Rodríguez y Cadle, 1990).

El objetivo del presente estudio es conocer la herpetofauna de la Zona Reservada Allpahuayo - Mishana en relación a algunos tipos de bosque, además de utilizar métodos de evaluación biológica rápida, en el marco del curso "Uso de inventarios biológicos para revelar patrones de distribución geográfica de especies en la selva baja amazónica", organizado por el proyecto Diversidad Biológica de la Amazonía Peruana, Perú - Finlandia (BIODAMAZ) $^{7}$, con el apoyo del Instituto de Investigaciones de la Amazonía Peruana (IIAP) y la Universidad Nacional de la Amazonía Peruana (UNAP). Asimismo, se buscó evaluar especies de anfibios (en particular Dendrobatidae) como indicadoras de ecosistemas generales.

\section{MATERIAL Y MÉTODO}

\section{1. Área de estudio}

El estudio se realizó en la Estación Biológica Allpahuayo (EBA) (358’9” S, 73²5’10” O), y en la Estación Experimental El Dorado (357'14” S, 73²4'70" O), ambas ubicadas en la Zona Reservada Allpahuayo Mishana (ZRAM), Loreto, Perú. La primera estación se localiza a la altura del km 26 de la carretera Iquitos Nauta, y es de propiedad del Instituto de Investigaciones de la Amazonía Peruana (IIAP), mientras que el segundo está a la altura del km 25 de la misma carretera en la estación El Dorado, que es de propiedad del Instituto Nacional de Investigaciones Agrarias (INIA). El muestreo herpetológico se desarrolló durante los días 18 al 22 noviembre del 2000.

La ZRAM presenta diversos tipos de bosque, siendo de particular importancia los bosques sobre arena blanca, localmente llamados varillales. Para el presente estudio se escogió tres tipos de bosque: varillal alto seco, varillal húmedo y un bosque de terrazas de la Formación Pebas (este último nombre es eventual y arbitrario, pues tomará otra denominación cuando se finalice la caracterización botánica y se relacione ésta con los tipos de suelo).

A continuación describimos de forma general los tres tipos de bosque donde se desarrolló este estudio:

1. El bosque de varillal seco se caracteriza por presentar una vegetación alta con árboles mayores de $3 \mathrm{~cm}$ de diámetro y hasta 10-25 m de altura; los árboles son rectos y delgados y los helechos son abundantes (Kalliola et al., 1993). El varillal seco presenta generalmente buen drenaje, por encontrarse en colinas altas con buena escorrentía de lluvias; además crece sobre un suelo de arena blanca, muy pobre en nutrientes.

2. El bosque de varillal húmedo presenta un suelo muy húmedo debido al drenaje deficiente, causado por un horizonte espódico impermeable, y se forman zonas fangosas y pantanosas. Se caracteriza por las abundantes

7 Proyecto Diversidad Biológica de la Amazonía Peruana (BIODAMAZ) es un convenio entre los gobiernos del Perú y de Finlandia ejecutado conjuntamente por el Instituto de Investigaciones de la Amazonía Peruana (IIAP) y por el consorcio finlandés formado por la empresa de consultorías ambientales, Biota BD Oy, y por la Universidad de Turku. 
bromelias (terrestres y epífitas), musgos, y la abundancia de la palmera "huasaí de varillal" (Euterpe catinga).

3. Finalmente, el bosque de terrazas de la Formación Pebas presenta un suelo franco-arenoso y es más fértil que ambos tipos de varillal.

Además, estos tres tipos de bosque presentaban un lecho de hojarasca bastante pobre, característico de muchas áreas de bosque amazónico, debido a la rápida descomposición y al aprovechamiento de nutrientes por las plantas, y por la influencia del clima húmedo tropical.

\subsection{Parcelas y transectos}

Para el trabajo de campo utilizamos lámparas frontales, bolsas plásticas, redes y gancho para serpientes. Para tener referencia de la ubicación de los lugares de muestreo utilizamos un GPS Garmin 12. Para medir algunos factores físicos utilizamos un termómetro/higrómetro electrónico. También fue utilizada, finalmente, una brújula Brunton, una cinta métrica de $50 \mathrm{~m}$, línea de pesca (de nylon), y cintas marcadoras para delimitar las parcelas y transectos.

La evaluación de la herpetofauna estuvo integrada por un equipo de seis personas. Durante el día fueron establecidas parcelas (Leaf-Litter Plots) para evaluar principalmente especies presentes en hojarasca.

Delimitamos dos parcelas de $20 \mathrm{~m}$ x $20 \mathrm{~m}$ en el varillal alto seco (P1 y P2), ambas ubicadas en una plataforma angosta en la cumbre plana de colinas de arenas blancas, con dosel de 15-20 m de altura. Estas áreas representativas fueron escogidas arbitrariamente, tratando de incluir áreas típicas de cada tipo de bosque. Asimismo, ubicamos otras dos parcelas de 20 m x 20 m en el bosque de terrazas de la Formación Pebas (P3 y P4), siguiendo criterios similares aplicados a las primeras, con dosel de 20-25 m.

El muestreo diurno de la herpetofauna consistió en revisar las parcelas en forma sistemática, removiendo el colchón de hojarasca y los restos de madera en descomposición. No se hizo muestreo de parcelas en el varillal húmedo (El Dorado), sino sólo de transectos lineales.

Durante la noche recorrimos transectos lineales, realizando registro visual y auditivo (RVA) de las especies, entre las 20.00 horas y las 01.00 horas, con ayuda de linternas frontales. Este método consistió en muestrear la herpetofauna a lo largo de fajas de $3 \mathrm{~m}$ de ancho y que variaron de longitud entre 750 y $1100 \mathrm{~m}$ en los diferentes lugares de muestreo. Este método permite muestrear la herpetofauna en diferentes estratos del bosque (especies de hojarasca y especies arbóreas).

El transecto ubicado en la trocha de plantas medicinales de la EBA (T1) tuvo una longitud de $1100 \mathrm{~m}$. Es una trocha ancha (3-4 m) que parte de la estación en dirección sur (Formación Pebas). El segundo transecto (T2) fue la trocha ecológica de la EBA, hasta el lote de frutales nativos, y tuvo una longitud de $980 \mathrm{~m}$. Es una trocha ancha ubicada en un bosque de terrazas de la Formación Pebas. El tercer transecto (T3) estuvo ubicado en la estación El Dorado, con una longitud de $750 \mathrm{~m}$. Es una trocha angosta $(0,8 \mathrm{~m})$, localizada en un varillal húmedo.

Fueron tomados los siguientes datos de los especímenes capturados: peso (medida con una balanza de precisión de 5 y 60 g, marca Pesola), SVL = longitud hocico-cloaca en anuros, y longitud total en otros grupos (utilizando un calibrador o vernier); además, fueron tomados datos de altura y del sustrato donde se encontraba cada especie. También se registró la temperatura ambiental y la humedad relativa.

La identificación taxonómica de los anfibios y reptiles se realizó con la ayuda de las siguientes guías de identificación: Dixon y Soini (1986), Duellman (1978), Rodríguez y Duellman (1994), y Duellman y Mendelson III (1995). La mayoría de los especimenes fueron fotografiados, con una cámara fotográfica Canon AE 1, y luego liberados. 


\subsection{Métodos de análisis}

Para evaluar la similitud y diferencia en la composición de especies entre los tipos de bosque estudiados utilizamos el coeficiente de similitud de Jaccard:

Siendo:

$$
\mathrm{Sj}=\frac{\mathrm{a}}{\mathrm{a}+\mathrm{b}+\mathrm{c}}
$$

$\mathrm{Sj}=$ Coeficiente de Similitud de Jaccard;

$\mathrm{a}=$ Número de especies compartidas entre dos lugares;

$\mathrm{b}=$ Número de especies en el primer lugar (varillal, $\mathrm{P} 1+\mathrm{P} 2$ );

$\mathrm{c}=$ Número de especies en el segundo lugar (terraza, P3 + P4).

Esta comparación sólo se realizó entre los bosques donde se trabajó con parcelas (bosque de varillal alto seco y de terrazas de la Formación Pebas). No se hizo la comparación entre transectos, porque éstos fueron de longitud y tiempo de muestreo variables y cruzaban diferentes tipos de bosque.

La abundancia relativa se calculó dividiendo el número de individuos de las especies más abundantes entre el número total de individuos encontrados en cada transecto. En el cálculo sólo se ha considerado a los individuos registrados mediante el método RVA.

\section{RESULTADOS}

Como resultado del trabajo de muestreo, en el cual invertimos un total de 195 horas-hombre, se registró un total de 66 especies, de las cuales 49 fueron anfibios (pertenecientes a 22 géneros) y 17 reptiles (14 géneros) (Tabla 1). A continuación comparamos la abundancia de individuos y riqueza de especies entre los diferentes tipos de bosque muestreados.

\subsection{Parcelas}

Para el análisis de las parcelas calculamos la densidad de individuos y riqueza de especies en cada uno de los dos tipos de bosque, para lo cual se juntaron los valores de las dos parcelas para cada tipo de bosque. Así, en el varillal seco de arena blanca se encontró un total de 16 individuos pertenecientes a 10 especies, con una densidad de 2 individuos $/ 100 \mathrm{~m}^{2}$. En el bosque de terrazas de la Formación Pebas se encontró un total de 40 individuos pertenecientes a 19 especies, con una densidad de 5 individuos $/ 100 \mathrm{~m}^{2}$, siendo esto más del doble de la densidad del bosque anterior (Figura 1). También el número de especies encontradas fue mayor en el bosque de terraza de Formación Pebas que en varillal seco (Figura 2).

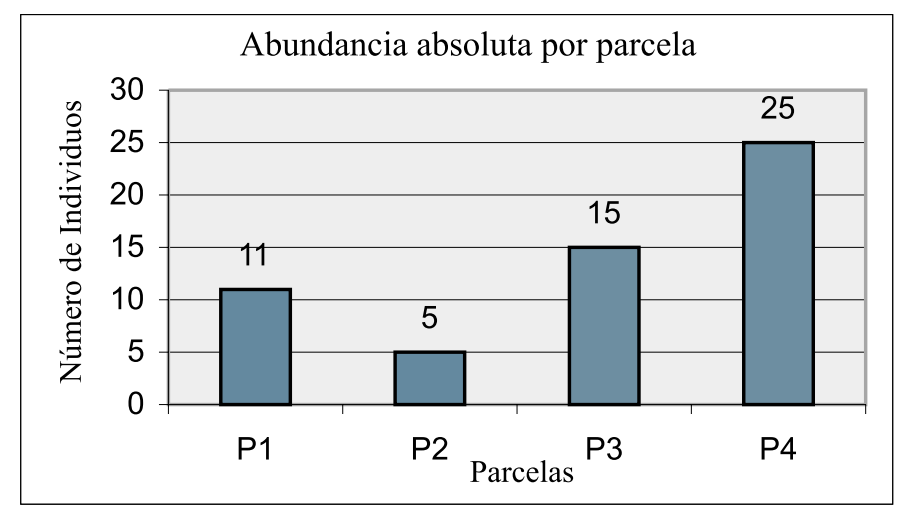

Figura 1. Abundancia absoluta por parcela. 


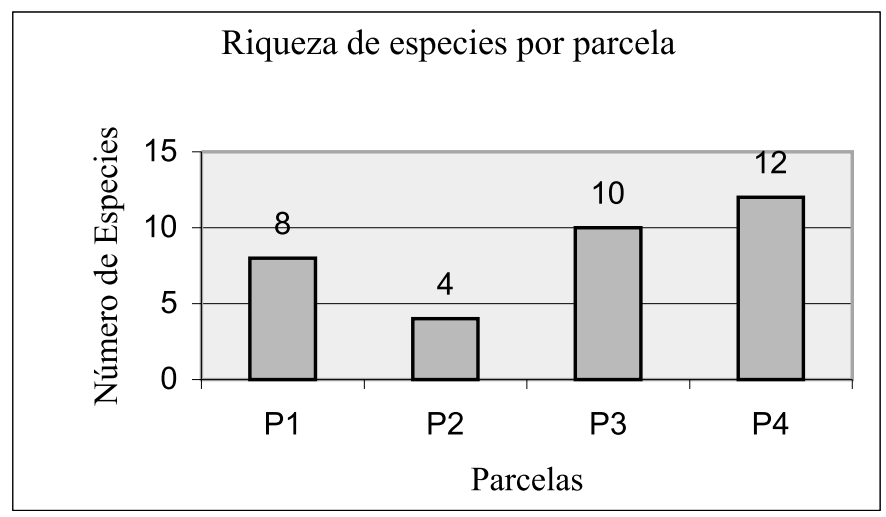

Figura 2. Riqueza de especies por parcela.

El número de especies compartidas entre el varillal y el bosque de Formación Pebas fue de cuatro (Bufo typhonius, Dendrobates reticulatus, Ischnocnema quixensis, y Syncope carvalhoi). El número de especies en el varillal fue 10, mientras que el número de especies en el bosque de Formación Pebas fue 19. Luego de aplicar el coeficiente de similitud de Jaccard $(\mathrm{Sj}=0.12)$, se encontró que los dos tipos de bosque comparten solo el $12 \%$ de las especies.

\subsection{Transectos}

Del número total de especies registradas en los transectos (43), el transecto T1 tuvo 65.1\%, y 53.5\% el T2, mientras que el T3 ubicado en el varillal húmedo registró solo 20.9\% (Figura 3).

Las especies con mayor número de individuos en T1 son B. typhonius (abundancia relativa =11,8\%), Hyla fasciata, H. lanciformis, S. carvalhoi (10.5\% cada uno), Adenomera sp. (7.9\%) e I. quixensis (6.6\%). En T2 las especies con mayor número de individuos son H. fasciata (10.8\%), Osteocephalus planiceps e I. quixensis (8.1\% cada uno). En cambio, Osteocephalus sp. presentó una abundancia relativa de $60 \%$ en T3 (Figura 4). Se excluye a los reptiles de este análisis debido al reducido número de registros logrados.

En general, se pudo notar en todos los transectos que algunas especies presentaban el mayor número de individuos, mientras que la mayoría de las 43 especies presentaban pocos individuos (Figura 4). Además, sólo tres especies, Phrynohyas resinifictrix, Bolitoglossa gr. altamazonica y Gonatodes humeralis, estuvieron presentes en los tres transectos (Tabla 1).

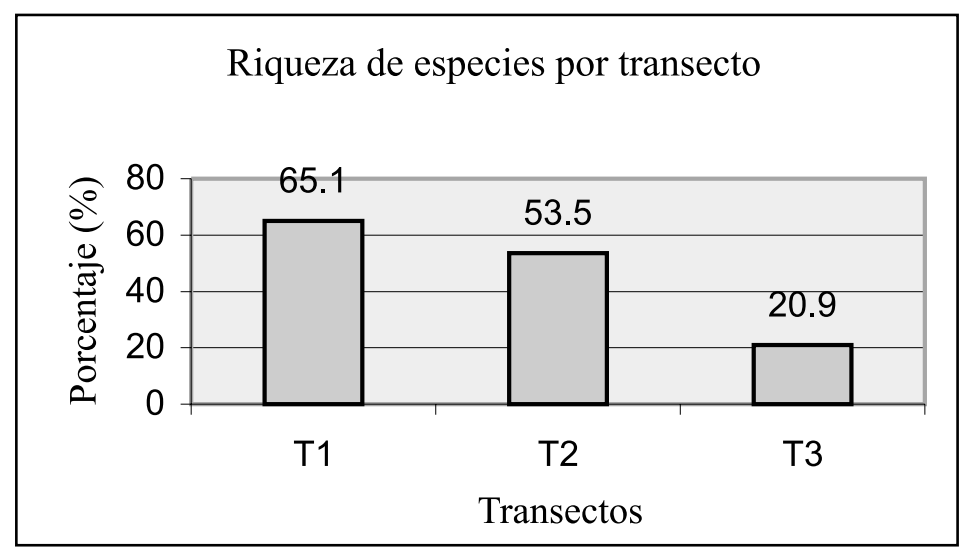

Figura 3. Riqueza de especies por transecto 


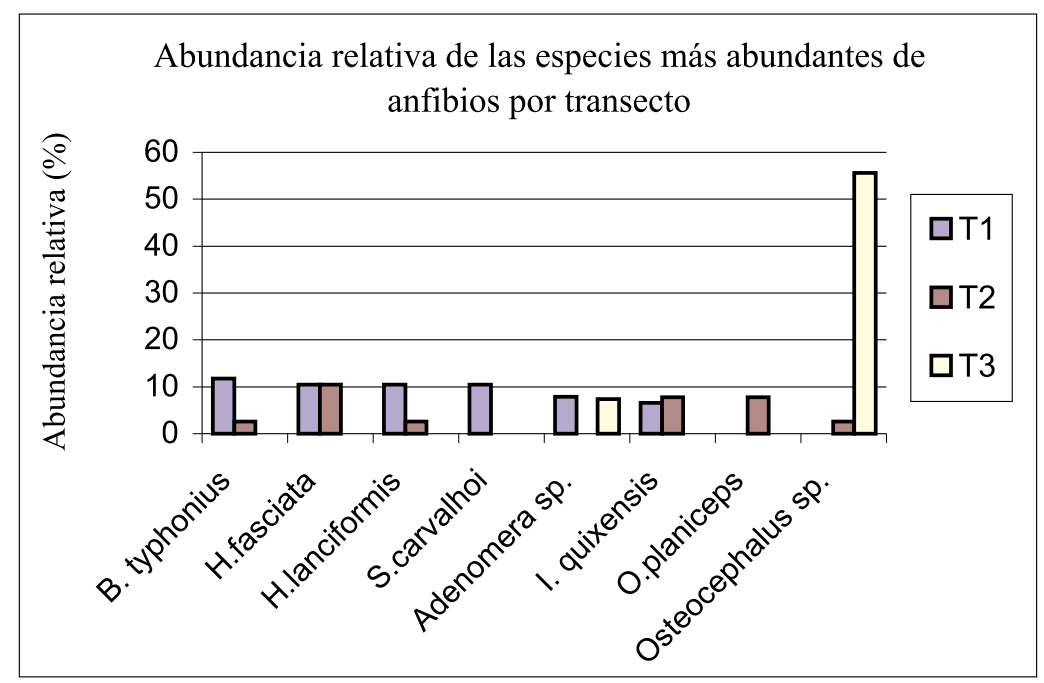

Figura 4. Abundancia relativa de las especies más abundantes de anfibios por transecto

\section{DISCUSIÓN}

En la presente evaluación, en la que se invirtió un total de 195 horas-hombre, con un equipo de seis personas que revisaron cuatro parcelas de muestreo de $20 \mathrm{~m}$ x $20 \mathrm{~m}$, y tres transectos lineales, logramos detectar 49 especies de anfibios y 17 especies de reptiles. Éstas incluyen 6 especies de anfibios como nuevos registros para la ZRAM, de las cuales tres especies (una Hyla y dos Eleutherodactylus) posiblemente serían nuevas para la Amazonía peruana. (Anexo 1).

Sin embargo, muchas especies de anfibios escuchadas en el dosel del bosque y en el estrato alto del sotobosque no fueron identificadas, debido a que aún no se conocen sus cantos.

De las especies de anfibios anteriormente ya conocidas de Allpahuayo y El Dorado, $67 \%$ fueron registradas en esta evaluación, mientras que de los reptiles solo $21 \%$ (pero en lagartijas 55\%), (Rivera, 1999; informe inédito IIAP), indicando que cualquier evaluación de este último grupo requiere un esfuerzo de muestreo mucho mayor.

Nuestros resultados indican que existe mayor abundancia y riqueza de especies en el bosque de terrazas de la Formación Pebas que en el bosque de varillal. Esto también concuerda con estudios realizados en plantas (Gentry, 1988), lo que probablemente está relacionado con una mayor riqueza de nutrientes en el suelo.

El análisis de similitud aplicado a parcelas indica que pocas especies son compartidas entre ambos tipos de bosque (12\%). Es probable que se compartan más especies entre ambos lugares, pero esto no se evidenció en el presente estudio. Por otro lado, la comparación de las abundancias relativas de las especies más abundantes entre los transectos puede servir para diferenciar tipos de bosque con características específicas. Por ejemplo, en el bosque de varillal húmedo se presentó una marcada dominancia de Osteocephalus sp., debido probablemente a la abundancia de plantas hospederas (Bromelias). Sin embargo, este resultado puede tener un sesgo importante debido a que en la única noche en que se recorrió el transecto en ese tipo de bosque hubo una garúa constante, previa a una lluvia fuerte, y la gran actividad de esa especie hizo relativamente fácil su localización a lo largo del transecto (sobre y dentro de axilas de bromelias). Por lo tanto, se considera necesario tener más réplicas en cada tipo de bosque, tanto para los transectos como para las parcelas.

Entre los km 25 y 27 de la carretera Iquitos - Nauta se encontró muchas ranas muertas por el tránsito de vehículos. Sorprendentemente, se registró una especie de Hyla, aparentemente nueva para la ciencia, cuando la lluvia caía fuerte sobre la pista de asfalto. Es importante también tomar en cuenta estos registros cuando se hace un inventario de especies de un lugar determinado. 
El resultado del muestreo referente a las especies indicadoras de la familia Dendrobatidae no tuvo mucho éxito. La densidad de especies del género Dendrobates fue sumamente baja por la falta casi total de plantas hospederas en los bosques alrededor de la Estación Biológica Allpahuayo, en cuatro parcelas de muestreo y a lo largo de los transectos, a excepción del transecto T3. Es evidente que las especies de Dendrobates están ausentes si faltan sus plantas hospederas principales, como las bromelias Aechmea y otras como Musacea y Araceae con fitotélmatas (cuerpos de agua sustentados por estructuras de plantas). Las únicas especies de Dendrobates encontradas en las parcelas fueron $D$. reticulatus y $D$. ventrimaculatus, durante una visita anterior a la trocha del transecto T3. D. amazonicus no fue registrado en nuestra evaluación, el cual es un habitante típico de bosques sobre arena blanca en el sector de Puerto Almendras, cerca de Iquitos.

En referencia a los Epipedobates, hemos detectado las especies usuales -E. femoralis y E. hahneli- en sus variantes locales. Colostethus "marchesianus" fue otro dendrobátido que siempre está relacionado con el grupo de las dos especies anteriores. La cuarta especie regional de la selva baja, Epipedobates trivittatus, está notoriamente ausente en la margen izquierda del Marañón - Amazonas, pero presente en la margen derecha y en la parte alta de la Reserva Nacional Pacaya - Samiria. Igualmente, E. trivittatus está ausente en los inventarios de la herpetofauna realizados en Ecuador (Duellman, 1978) y en San Jacinto, río Tigre (Duellman y Mendelson III, 1995).

El análisis preliminar de los cantos indica grandes diferencias entre las variantes de éstos en E. hahneli, si comparamos el canto con el de las ranas de la misma especie de la localidad tipo en Yurimaguas. Los individuos de E. hahneli de la ZRAM tienen un canto de cuatro pulsos muy acelerados, altos y rápidos. Del mismo modo, el canto de Colostethus "marchesianus" de la ZRAM no concuerda con el canto de las ranas presentes en el sector de Yurimaguas (R. Schulte, obs. pers.). Estas diferencias serán investigadas posteriormente.

El análisis de especies de Hylidae es una buena medida para detectar diferencias en los ecosistemas locales, mientras que mediante el análisis de los dendrobátidos se logra detectar refugios más grandes y antiguos si usamos el análisis de las variantes. Por ejemplo, faltan las ranas de D. flavovittatus en la margen izquierda del Marañón - Amazonas, mientras que D. reticulatus no está presente en el lado derecho. Las únicas especies presentes en las dos orillas son D. ventrimaculatus (variante amarillo) y D. quinquevittatus (R. Schulte, obs. pers.).

\section{AGRADECIMIENTOS}

Nuestro agradecimiento al proyecto BIODAMAZ Perú - Finlandia por la oportunidad de realizar este estudio.

\section{BIBLIOGRAFÍA}

BERGER, L.; SPEARE, R.; DASZAK, P.; GREEN, D.E.; CUNNINGHAM, A.A.; GOGGIN, C.L.; SLOCOMBE, R.; RAGAN, M.; HYATT, A.D.; MCDONALD, K.R.; HINES, H.B.; LIPS, K.R.; MARANTELLI, G.; PARKES, H. 1989. Chytridiomycosis causes amphibian mortality associated with population declines in the rain forests of Australia and Central America. Proc. Natl. Acad. Sci. USA 95: 9031-9036.

CRUMP, M.L. 1974. Reproductive strategies in a tropical anuran community. Misc. Publ. Mus. Nat. Hist. Univ. Kansas 61: 1-68.

DIXON, J.R.; SOINI, P. 1986. The reptiles of the upper Amazon basin, Iquitos Region, Peru. 2nd Edition. Milwaukee Public Museum, Milwaukee, Wisconsin, EE.UU.

DONNELLY, M.A.; CRUMP, M.L. 1998. Potential effects of climate change on two Neotropical amphibian assemblages. Climatic Change 39: 541-561. 
DUELLMAN, W.E. 1978. The biology of an equatorial herpetofauna in Amazonian Ecuador. Misc. Publ. Mus. Nat. Hist. Univ. Kansas 65: 1-352.

DUELLMAN, W.E. 1990. Herpetofaunas in Neotropical rainforests: Comparative composition, history and resource use. En: A. H. Gentry (ed.). Four Neotropical Rainforests. New Haven, CT y Londres. 455$505 \mathrm{pp}$.

DUELLMAN, W.E.; SALAS, A.W. 1991. Annotated checklist of the amphibians and reptiles of Cuzco Amazonico, Peru. Occas. Pap. Mus. Nat. Hist. Univ. Kansas 143: 1-13.

DUELLMAN, W.E.; MENDELSON III, J.R. 1995. Amphibians and reptiles from northern Departamento Loreto, Peru: Taxonomy and Biogeography. Univ. Kansas Sci. Bull. 55 (10): 329-376.

GENTRY, A. H. 1988. Tree species richness of upper Amazonian forest. Proc. Nat. Acad. Sci. 85: 156-159.

KALLIOLA, R.; PUHAKKA, M.; DANJOY, W. (eds.). 1993. Amazonía peruana. Vegetación húmeda tropical en el llano subandino. Proyecto Amazónica de la Universidad de Turku y Oficina Nacional de Evaluación de Recursos Naturales. Jyväskylä, Finlandia. 265 pp.

RODRÍGUEZ, L.O. 1992. Structure et organisation du peuplement d'Anoures de Cocha Cashu, Parc National Manu, Amazonie Péruvienne. Rev. Ecol. (Terre Vie) 47: 151-197.

RODRÍGUEZ, L.O.; CADLE, J.E. 1990. A Preliminary overview of the herpetofauna of Cocha Cashu, Manu National Park, Peru. En: A. H. Gentry (ed.). Four Neotropical Rainforests. Yale University Press, New Haven, CT y Londres. 410-425 pp.

RODRÍGUEZ, L.O.; DUELLMAN, W. 1994. Guide to the frogs of the Iquitos Region Amazonian Peru. University of Kansas Press. Kansas, EE.UU. 80 pp.

VIAL, J.L.; SAYLOR, L. 1993. The status of amphibian populations: A compilation and analysis. UICN/SSC DAPTF Working Doc. 1: 1-98.

WAKE, D.B.; MOROWITZ, H.G. 1991. Declining amphibian populations. A global phenomenon? Findings and recommendations. Alytes 9: 33-42. 
Anexo 1. Anfibios y reptiles registrados en las Estaciones Allpahuayo - IIAP y El Dorado - INIA (Zona Reservada Allpahuayo - Mishana ).

\begin{tabular}{|c|c|c|c|c|c|c|c|c|c|c|}
\hline \multirow{3}{*}{ TAXON } & \multirow{2}{*}{\multicolumn{2}{|c|}{$\begin{array}{l}\text { Tipo de } \\
\text { registro }\end{array}$}} & \multicolumn{7}{|c|}{$\mathrm{N}^{\mathrm{o}}$ de individuos registrados } & \multirow[t]{3}{*}{ Carret. } \\
\hline & & & & ansec & & & & las & & \\
\hline & $\mathrm{V}$ & $\mathrm{A}$ & $\mathrm{T} 1$ & $\mathrm{~T} 2$ & T3 & P1 & $\mathrm{P} 2$ & P3 & $\mathrm{P} 4$ & \\
\hline AMPHIBIA & & & & & & & & & & \\
\hline ANURA & & & & & & & & & & \\
\hline Bufonidae & & & & & & & & & & \\
\hline Bufo marinus & + & + & $*$ & & & & & & & + \\
\hline Bufo typhonius & + & & 9 & 1 & & 2 & & 2 & 8 & \\
\hline Centrolenidae & & & & & & & & & & \\
\hline Cochranella ritae $* *$ & + & + & 1 & & & & & & & \\
\hline DENDROBATIDAE & & & & & & & & & & \\
\hline Colostethus marchesianus & + & & & & & & & 2 & 2 & \\
\hline Dendrobates reticulatus & + & & 1 & 1 & & 2 & 1 & 1 & & \\
\hline Dendrobates ventrimaculatus & + & & & & 1 & & & & & \\
\hline Epipedobates femoralis & + & + & 2 & & & & & & & \\
\hline Epipedobates hahneli & + & + & 3 & & 1 & 1 & & & & \\
\hline Hylidae & & & & & & & & & & \\
\hline Hyla calcarata & + & + & 1 & & & & & & & \\
\hline Hyla fasciata & + & + & 8 & 4 & & 1 & & & & \\
\hline Hyla geographica & + & & $*$ & & & & & & & \\
\hline Hyla lanciformis & + & + & 8 & 1 & & & & & & + \\
\hline Hyla leucophyllata & + & + & 2 & & & & & & & + \\
\hline Hyla punctata & + & + & 2 & & & & & & & \\
\hline Hyla sp.A ?** & + & & & & & & & & & + \\
\hline Nyctimantis rugiceps & & + & $*$ & & & & & & & \\
\hline Osteocephalus cabrerai & + & + & & 2 & & & & & 1 & \\
\hline Osteocephalus planiceps & + & & & 3 & & 1 & & & & \\
\hline Osteocephalus sp. & + & & & 1 & 15 & & & & & \\
\hline hrynohyas resinifictrix & & + & $*$ & $*$ & $*$ & & & & & \\
\hline Phrynohyas venulosa & + & & & & & & & & & + \\
\hline Phyllomedusa tomoptera & + & & & & 1 & & & & & + \\
\hline Phyllomedusa vaillanti & + & & 1 & 1 & & & & & & \\
\hline Scinax garbei & + & & & 1 & & & & & & + \\
\hline Scinax rubra & + & & 2 & & & & & & & + \\
\hline LEPTODACTYLIDAE & & & & & & & & & & \\
\hline Adenomera sp & + & + & 6 & & 2 & & & 1 & 1 & \\
\hline Eleutherodactylus acuminatus & + & & $*$ & 2 & & & & & & \\
\hline Eleutherodactylus carvalhoi & + & & 1 & 2 & & & & & 2 & \\
\hline Eleutherodactylus diadematus *** & + & & $*$ & & & & & & & \\
\hline Eleutherodactylus lanthanites & + & & 1 & & & & & & 2 & \\
\hline Eleutherodactylus ockendeni & + & & 1 & 2 & & 1 & & & & \\
\hline Eleutherodactylus peruvianus & + & & & 1 & & & & & & \\
\hline Eleutherodactylus sp. A & + & & $*$ & 1 & & & & & & \\
\hline Eleutherodactylus sp. B & + & & & & 1 & & & & & \\
\hline
\end{tabular}




\begin{tabular}{|c|c|c|c|c|c|c|c|c|c|c|}
\hline \multirow{3}{*}{ TAXON } & \multirow{2}{*}{\multicolumn{2}{|c|}{$\begin{array}{l}\text { Tipo de } \\
\text { registro }\end{array}$}} & \multicolumn{7}{|c|}{$\mathrm{N}^{\circ}$ de individuos registrados } & \multirow[t]{3}{*}{ Carret. } \\
\hline & & & \multicolumn{3}{|c|}{ Transectos } & \multicolumn{4}{|c|}{ Parcelas } & \\
\hline & $\mathrm{V}$ & $\mathrm{A}$ & $\mathrm{T} 1$ & $\mathrm{~T} 2$ & $\mathrm{~T} 3$ & P1 & $\mathrm{P} 2$ & $\mathrm{P} 3$ & $\mathrm{P} 4$ & \\
\hline Eleutherodactylus sp. D** & + & & 1 & & & & & & & \\
\hline Eleutherodactylus sp. E ** & + & & 1 & 1 & & & & 2 & & \\
\hline Eleutherodactylus sulcatus & + & & & & & 1 & & & & \\
\hline Ischnocnema quixensis & + & & 5 & 3 & & & 1 & & 2 & \\
\hline Leptodactylus pentadactylus & + & + & $*$ & & & & & & & \\
\hline Leptodactylus rhodomystax & + & & & & & & & & 1 & \\
\hline Leptodactylus stenodema & & + & $*$ & & & & & & & \\
\hline Leptodactylus wagneri & + & & & 1 & & & & & & \\
\hline Lithodytes lineatus $* *$ & + & & & & & & & & & + \\
\hline Phyllonastes myrmecoides & + & & & & & & & & 3 & \\
\hline Physalaemus petersi & + & & & 2 & & & & & & \\
\hline MICROHYLIDAE & & & & & & & & & & \\
\hline Chiasmocleis bassleri & + & & & & & & & 1 & & \\
\hline Hamptophryne boliviana & & + & $*$ & & & & & & & \\
\hline Syncope carvalhoi & + & & 8 & & & 2 & 2 & 3 & & \\
\hline CAUDATA & & & & & & & & & & \\
\hline Plethodontidae & & & & & & & & & & \\
\hline Bolitoglossa gr. altamazonica & + & & $*$ & 2 & 2 & & & & & \\
\hline REPTILIA SQUAMATA & & & & & & & & & & \\
\hline SAURIA GEKKONIDAE & & & & & & & & & & \\
\hline Gonatodes humeralis & + & & 2 & $*$ & 1 & & 1 & & & \\
\hline Pseudogonatodes guianensis & + & & & & & & & & 1 & \\
\hline Thecadactylus rapicaudus & + & & 1 & & & & & & & \\
\hline GYMNOPHTHALMIDAE & & & & & & & & & & \\
\hline Arthrosaura reticulata & + & & & & & & & & 1 & \\
\hline Iphisa elegans & + & & & & & & & 1 & & \\
\hline Neusticurus ecpleopus & + & & & $*$ & & & & & & \\
\hline Prionodactylus argulus & + & & 1 & $*$ & & & & & 1 & \\
\hline POLYCHROTIDAE & & & & & & & & & & \\
\hline Anolis bombiceps & + & & & 2 & & & & 1 & & \\
\hline Anolis fuscoauratus & + & & 3 & & 3 & & & & & \\
\hline Anolis ortonii & + & & 1 & & & & & & & \\
\hline Anolis transversalis & + & & & & $*$ & & & & & \\
\hline SCINCIDAE & & & & & & & & & & \\
\hline Mabuya nigropunctata & + & & 1 & & & & & & & \\
\hline TEIIDAE & & & & & & & & & & \\
\hline Ameiva ameiva & + & & $*$ & & & & & & & \\
\hline Kentropyx pelviceps & + & & & 1 & & & & 1 & & \\
\hline TROPIDURIDAE & & & & & & & & & & \\
\hline Stenocercus fimbriatus & + & & 3 & 2 & & & & & & \\
\hline SERPENTES & & & & & & & & & & \\
\hline COLUBRIDAE & & & & & & & & & & \\
\hline Chironius fuscus & + & & 1 & & & & & & & \\
\hline Imantodes cenchoa & + & & & 1 & & & & & & \\
\hline
\end{tabular}

